\title{
BALlOON KyphOplasty fOR Vertebral Compression Fracture Using a Unilateral Balloon Tamp Via a UnI-PEdicular ApProach: TECHNICAL NOTE
}

\author{
Brian L. Hoh, MD, James D. Rabinov, MD, Johnny C. Pryor, MD, and Joshua A. Hirsch, MD
}

\begin{abstract}
Objective: Percutaneous balloon kyphoplasty, like percutaneous vertebroplasty is a therapeutic intervention for painful osteoporotic vertebral body compression fracture. The procedure involves placement of bilateral inflatable balloon tamps in the fractured vertebral body via a bilateral transpedicular or bilateral extra-pedicular approach. We describe performance of balloon kyphoplasty using a unilateral, single, balloon tamp via a unilateral transpedicular approach. The advantages of a unilateral approach are reducing the risk, albeit low, of pedicle fracture, medial transgression of the pedicle and/or transgression into the spinal canal, nerve injury, cement extravasation along
\end{abstract}

Percutaneous balloon kyphoplasty, like percutaneous vertebroplasty, is a therapeutic intervention performed for painful osteoporotic vertebral compression fracture. This procedure differs from conventional vertebroplasty in that an inflatable balloon bone tamp (Kyphon Inc., Santa Clara, CA) is advanced into the fractured vertebral body, the balloon is inflated to reduce the fracture and elevate the endplates, and balloon deflation produces a cavity into which bone cement, usually polymethylmethacrylate, can be injected. Several groups have reported favorable clinical and radiographic results using this technique (1-4).

The balloon kyphoplasty procedure consists of insertion of bilateral inflatable balloon tamps either via a bilateral transpedicular approach, bilateral parapedicu-

From Endovascular Neurosurgery and Interventional Neuroradiology, Neurosurgical Service and Department of Radiology, Massachusetts General Hospital, Harvard Medical School, Boston, MA. Address Correspondence: Joshua A. Hirsch, MD, Massachusetts General Hospital, Gray 289, 55 Fruit Street, Boston, MA 02114.

E-mail: jahirsch@partners.org

Funding: There was no funding in preparation of this manuscript.

Conflict of Interest: None the cannula tract, and spinal epidural hematoma. Additionally, operative and anesthesia time is reduced, as well as the costs of balloon tamps, cannulas, and needles.

Case Illustration: An 83-year-old woman with osteoporosis presented with severe lower thoracic back pain which occurred when she bent over to lift a heavy box. The pain was reproducible on palpation of the T-11 spinous process. A spine MRI with STIR (short tau inversion recovery) sequence demonstrated a subacute T-11 vertebral body compression fracture with associated edema. A T-11 balloon kyphoplasty was performed using a unilateral inflatable bal- loon tamp via a unilateral transpedicular approach. The patient reported immediate relief of pain and improvement of visual analog score (VAS) for pain from preoperative 10 to postoperative 2 . She was able to ambulate postoperatively whereas preoperatively she was inhibited by pain.

Conclusion: Balloon kyphoplasty can be performed using a unilateral balloon tamp via a unilateral pedicular approach. The key is a medial needle trajectory with a final balloon position in the midline of the vertebral body.

Keywords: Compression fracture, kyphoplasty, osteoporosis, minimally invasive, pain, vertebroplasty. lar (achieving entry at the costochondral junction) or a bilateral extra-pedicular approach. We describe performance of balloon kyphoplasty with a unilateral balloon tamp via a unilateral transpedicular approach. A unilateral approach offers the advantages of reducing the risk, albeit low, of pedicle fracture, medial transgression of the pedicle and/or transgression into the spinal canal, nerve injury, cement extravasation along the cannula tract, and spinal epidural hematoma. Additionally, operative and anesthesia time is reduced, as well as potentially, the costs of balloon tamps, cannulas, and needles.

\section{Case lllustration}

An 83-year-old woman with osteoporosis presented with severe lower thoracic back pain of several weeks, which she reported had a clear onset at the time she was bending over and lifting a heavy box of curtains. The back pain was refractory to medical therapy and rendered her unable to ambulate. She was admitted to the hospital for pain control. She denied radicular symptoms and/or bowel or bladder dysfunction. She had associated diagnoses of stable abdominal aortic aneurysm, chronic obstructive pulmonary disease, coronary artery disease, hypertension, and diverticulosis. Her medications included Fosamax, calcitonin spray, atenolol, hydrochlorothiazide, morphine, fluticasone inhaler, albuterol/ipratropium inhaler. On physical examination, she is a thin elderly woman with severe kyphotic deformity. Exquisite pain was reproducible on palpation of the T-11 spinous process. Otherwise, there were no radicular findings and her neurological examination was normal with normal motor strength, reflexes, sensation, and rectal tone.

A spine magnetic resonance imaging (MRI) study was performed which included T1-weighted, T2-weighted, and STIR (short tau inversion recovery) sequences. These demonstrated a subacute T-11 vertebral compression fracture with associated edema (Fig. 1).

Percutaneous T-11 balloon kyphoplasty was performed using a unilateral inflatable balloon tamp via a unilateral transpedicular approach. Briefly, after performance of general endotracheal anesthesia and administration of a pre-procedure dose of intravenous cefazolin, the patient was carefully positioned prone on the fluoroscopy table in a NEUROSTAR 


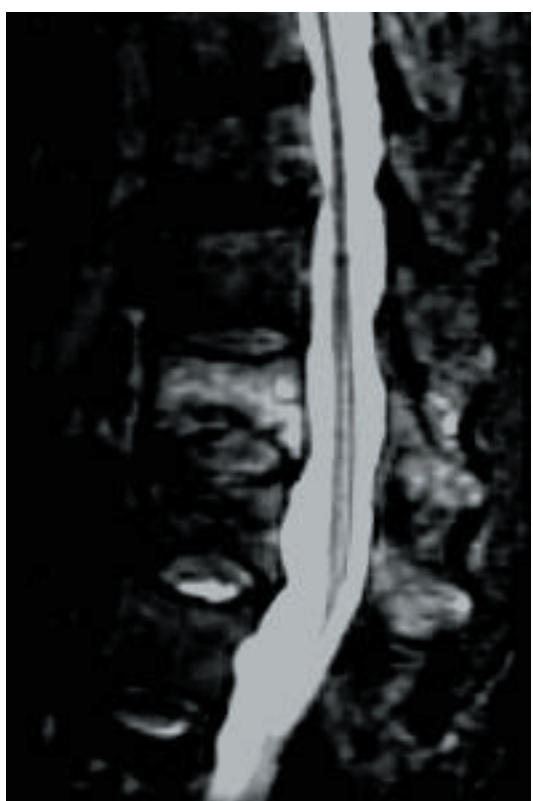

Fig 1. Spine magnetic resonance imaging (MRI) study with short tau inversion recovery (stir) sequence demonstrating subacute $t-11$ vertebral body compression fracture with associated edema. biplane angiography unit (Siemens, Munich, Germany). Using sterile technique, subcutaneous local $1 \%$ lidocaine was administered at the planned needle entry site for the left T-11 pedicle, which was localized by fluoroscopy. A small skin incision was made and an 11-guage needle was advanced through the left T-11 pedicle into the T-11 vertebral body utilizing intermittent biplane fluoroscopy. Special attention was made to performing a medial trajectory of the needle for a final midline destination of the needle tip in the vertebral body. The needle was exchanged for a working cannula over a K-wire through which a drill trocar was advanced creating a channel for the balloon (Fig. 2). The drill trocar was removed and the inflatable balloon tamp was advanced into the anterior one-third of the vertebral body under fluoroscopy. The balloon was inflated under fluoroscopy to $100 \mathrm{psi}$ and $4 \mathrm{~mL}$ of contrast (Figure 3). The balloon was deflated and removed. Polymethylmethacrylate (Parallax Medical, Mountain View, CA) was prepared with barium sulfate and when satisfactory consistency was achieved, was injected under fluoroscopy into the cavity in the vertebral body created by the balloon tamp (Fig. 4). A total of $5 \mathrm{~mL}$ of cement was administered which produced an excellent filling of the vertebral body cavity. All instrumentation was removed at the end of the procedure.

The patient reported immediate pain relief. Her self-reported visual analog score (VAS) for pain improved from a preoperative score of 10 to a postoperative score of 2 . She was able to ambulate whereas preoperatively she was inhibited by pain.

\section{DisCUSSION}

Percutaneous balloon kyphoplasty for painful osteoporotic vertebral body compression fractures has been described by several groups with good clinical and radiographic outcomes (1-4). Description of the procedure involves insertion of bilateral balloon tamps via a bilateral transpedicular or bilateral extra-pedicular approach. We describe performance of balloon kyphoplasty using a single unilateral balloon tamp via a unilateral transpedicular approach.

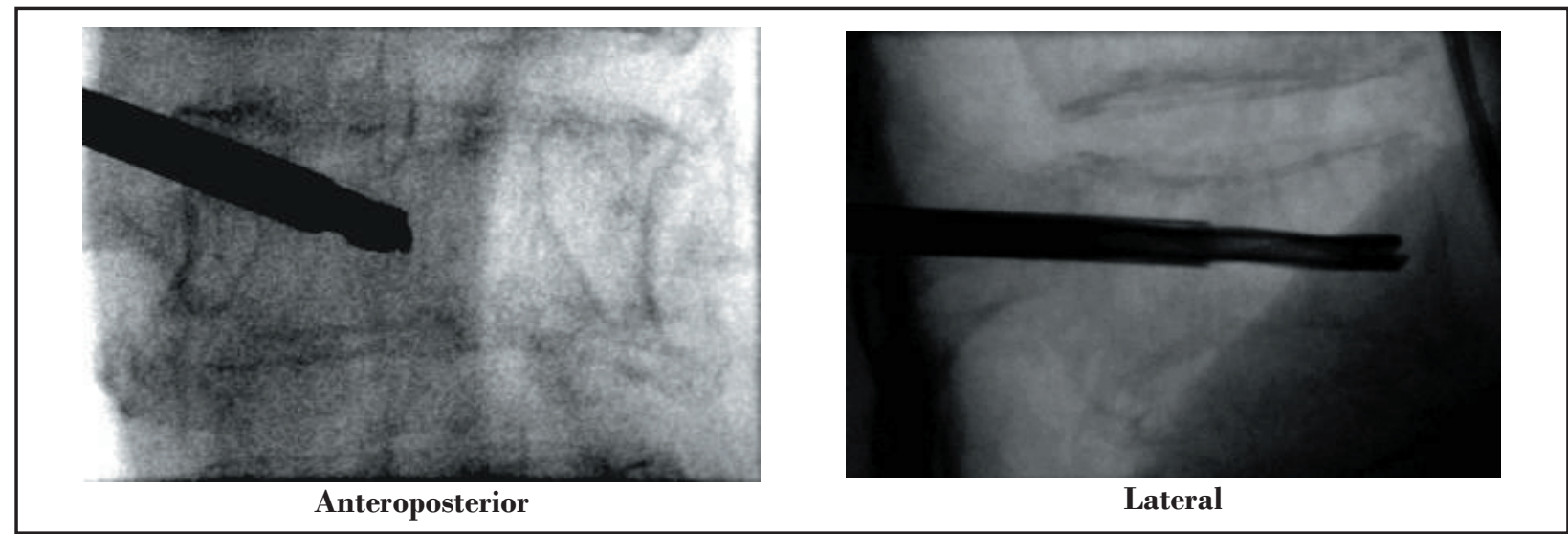

Fig 2. Lateral fluoroscopic image of medially directed working cannula and drill trocar.

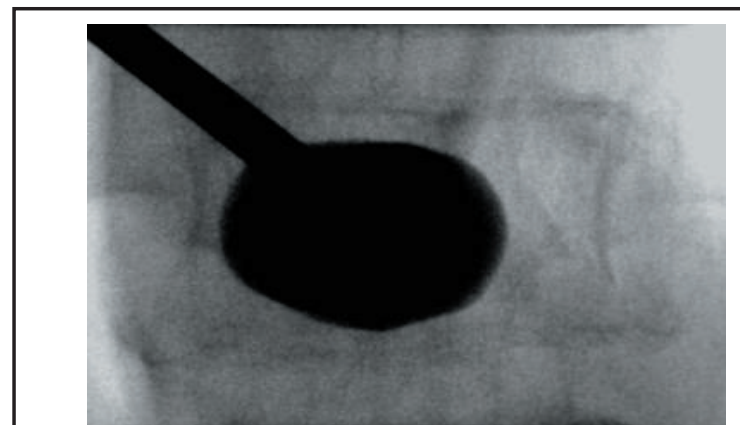

Anteroposterior

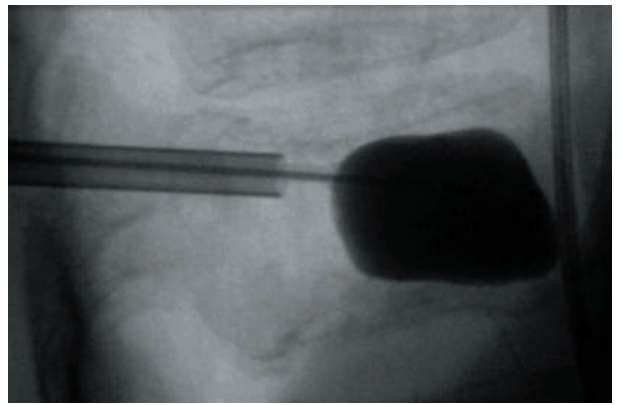

Lateral

Fig 3. Fluoroscopic image of inflated balloon tamp in the midline of the fractured vertebral body. 


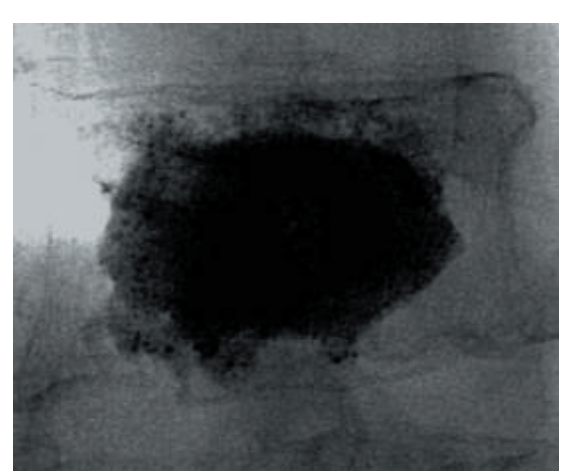

Anteroposterior

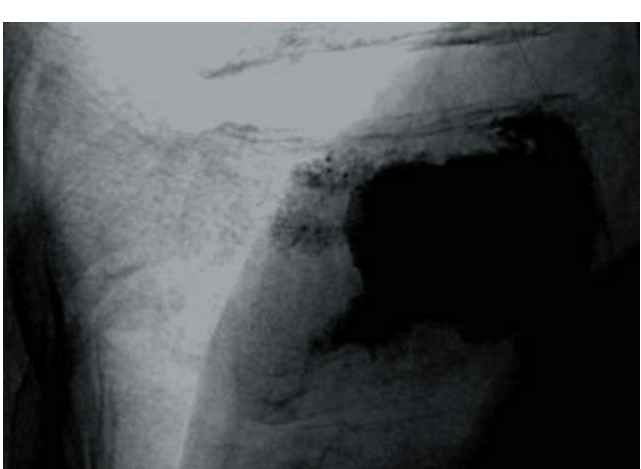

Lateral

Fig 4. Lateral fluoroscopic image of polymethylmethacrylate filling the cavity within the fractured vertebral body.

The rationale for the conventional use of bilateral balloons is to achieve adequate endplate elevation with two balloons and to create a large enough cavity for maximal cement filling. With conventional needle trajectories in a transpedicular or extrapedicular approach, the balloon tamps remain ipsilateral, thus necessitating bilateral balloons to cover the expanse of the vertebral body. In the procedure, we describe, we perform a medially-directed trajectory with the final destination of the balloon in the midline of the vertebral body. A midline-positioned balloon can be inflated to create a large enough cavity in the midline of the vertebral body. We have been satisfied with the extent of bone cement filling and elevation of endplates.

The advantages of a unilateral balloon tamp are reducing the risk, albeit low, of pedicle fracture, medial transgression of the pedicle and/or transgression into the spinal canal, nerve injury, cement extravasation along the cannula tract, and spinal epidural hematoma. In Coumans et al (2) a large series of $188 \mathrm{ky}-$ phoplasty procedures, there were only five cases of cement extravasation, however, three were along the cannula tract. This would be reduced by one-half if unilateral rather than bilateral cannulas were placed. Additionally, operative and anesthesia time is reduced, as well as the costs of balloon tamps, cannulas, and needles. In the procedure we describe, the time of patient entry into the angiography suite to time of patient departure (including time required for general anesthesia induction at the beginning of the procedure, and reversal of general anesthesia at the end of the procedure) was 90 minutes. Typical- ly, when we have performed balloon kyphoplasty with bilateral balloons, the total procedure time is 120 minutes.

The procedure was well tolerated by the patient with excellent clinical and radiographic result which we believe is comparable to balloon kyphoplasty using bilateral balloons. No study has directly compared balloon kyphoplasty to vertebroplasty, and we do not attempt to do so here.

In the event that balloon kyphoplasty is attempted using a unilateral balloon and the surgeon is unsatisfied with the balloon position or the extent of balloon inflation or cavity created, a second contralateral balloon can be placed using the conventional technique. It is thus our belief now that, except in rare circumstances where we know preoperatively that bilateral balloons will be needed because of unusual pathologic anatomy, that we always attempt a unilateral approach with the backup option of placing a second balloon if unsatisfied with the single balloon. The key to the unilateral approach is the medial trajectory of the needle and the final midline position of the balloon.

\section{CONCLUSION}

We describe performance of percutaneous balloon kyphoplasty for osteoporotic vertebral body compression fracture using a unilateral inflatable balloon tamp via a unilateral transpedicular approach. The key to the approach is a medial trajectory with a final position of the balloon in the midline of the vertebral body.

\section{Author Affiliation: \\ Brian L. Hoh, MD}

Endovascular Neurosurgery, Interventional Neuroradiology, and Neurosurgical Service

Massachusetts General Hospital

55 Fruit Street

Boston, MA 02114

E-mail:bhoh@partners.org

\section{James D. Rabinov MD}

Endovascular Neurosurgery and Interventional Neuroradiology

Massachusetts General Hospital

55 Fruit Street

Boston, MA 02114

\section{Johnny C. Pryor, MD}

Director, Endovascular Neurosurgery and Interventional Neuroradiology

Massachusetts General Hospital

55 Fruit Street

Boston, MA 02114

\section{Joshua A. Hirsch, MD}

Director, Endovascular Neurosurgery and Interventional Neuroradiology

Massachusetts General Hospital

55 Fruit Street

Boston, MA 02114

E-mail: jahirsch@partners.org

\section{REFERENCES}

1. Lieberman IH, Dudeney S, Reinhardt MK et al. Initial outcome and efficacy of "kyphoplasty" in the treatment of painful osteoporotic vertebral compression fractures. Spine 2001; 26:1631-1638. 
2. Coumans JV, Reinhardt MK, Lieberman IH. 3. Kyphoplasty for vertebral compression fractures: 1-year clinical outcomes from a prospective study. J Neurosurg 2003; 99:44-50.
Ledlie JT, Renfro M. Balloon kyphoplasty: One-year outcomes in vertebral body height restoration, chronic pain, and activity levels. J Neurosurg 2003; 98:36-42.
4. Garfin SR, Yuan HA, Reiley MA. New technologies in spine: Kyphoplasty and vertebroplasty for the treatment of painful osteoporotic compression fractures. Spine 2001; 26:1511-1515. 